\title{
Black Hole Entropy Quantization
}

\author{
Alejandro Corichi, ${ }^{1,2,3, *}$ Jacobo Díaz-Polo, ${ }^{4}$ and Enrique Fernández-Borja ${ }^{5}$ \\ ${ }^{1}$ Instituto de Matemáticas, Unidad Morelia, Universidad Nacional Autónoma de México, UNAM-Campus Morelia, \\ A. Postal 61-3, Morelia, Michoacán 58090, Mexico \\ ${ }^{2}$ Instituto de Ciencias Nucleares, Universidad Nacional Autónoma de México, A. Postal 70-543, México D.F. 04510, Mexico \\ ${ }^{3}$ Institute for Gravitational Physics and Geometry, Physics Department, Pennsylvania State University, \\ University Park, Pennsylvania 16802, USA \\ ${ }^{4}$ Departamento de Astronomía y Astrofísica, Universidad de Valencia, Burjassot-46100, Valencia, Spain \\ ${ }^{5}$ Departamento de Física Teórica and IFIC, Centro Mixto Universidad de Valencia-CSIC. Universidad de Valencia, \\ Burjassot-46100, Valencia, Spain
}

(Received 3 October 2006; published 4 May 2007)

\begin{abstract}
Ever since the pioneering works of Bekenstein and Hawking, black hole entropy has been known to have a quantum origin. Furthermore, it has long been argued by Bekenstein that entropy should be quantized in discrete (equidistant) steps given its identification with horizon area in (semi-)classical general relativity and the properties of area as an adiabatic invariant. This lead to the suggestion that the black hole area should also be quantized in equidistant steps to account for the discrete black hole entropy. Here we shall show that loop quantum gravity, in which area is not quantized in equidistant steps, can nevertheless be consistent with Bekenstein's equidistant entropy proposal in a subtle way. For that we perform a detailed analysis of the number of microstates compatible with a given area and show consistency with the Bekenstein framework when an oscillatory behavior in the entropy-area relation is properly interpreted.
\end{abstract}

DOI: 10.1103/PhysRevLett.98.181301

PACS numbers: 04.70.Dy, 04.60.Pp

Black hole entropy is one of the most intriguing constructs of modern theoretical physics. On the one hand, it has a correspondence with the black hole horizon area through the laws of (classical) black hole mechanics. On the other hand, it is assumed to have a quantum statistical origin given that the proper identification between entropy and area $S=A / 4 \ell_{p}^{2}$ came only after an analysis of quantum fields on a fixed background [1].

Furthermore, it has long been argued by Bekenstein that the proportionality between entropy and area, for large, classical black holes, can be justified from the adiabatic invariance properties of horizon area when subject to different scenarios (see $[2,3]$ for a review). Further heuristic quantization arguments lead to the suggestion that area, when quantized, should have a discrete, equidistant spectrum in the large horizon limit [3],

$$
A=\alpha \ell_{p}^{2} n,
$$

with $\alpha$ a parameter and $n$ integer. The relation between area and entropy that one expects to encounter in the large horizon radius is then extrapolated to the full spectrum. This would imply that entropy too would have a discrete spectrum, a property that might also be expected if entropy is to be associated with (the logarithm of) the number of microstates compatible with a given macrostate. When this condition is imposed, then the area is expected to have an spectrum of the form

$$
A=4 \ell_{p}^{2} \ln (k) n,
$$

with $k$ and $n$ integers [4]. Even when appealing and physi- cally well motivated, these arguments remain somewhat heuristic and have no detailed microscopic quantum gravity formalism to support them.

A quantum canonical description of black holes that has offered a detailed description of the quantum horizon degrees of freedom is given by loop quantum gravity (LQG) [5]. This formalism allows the inclusion of several matter couplings (including nonminimal couplings) and black holes far from extremality, in four dimensions. There is no restriction in the values of the matter charges. The approach uses as a starting point isolated horizon (IH) boundary conditions at the classical level, where the interior of the black hole is excluded from the region under consideration. The quantum degrees of freedom are excited when a spin network - a collection of edges with "spin" labels $j_{i}$ and vertices - pierces the horizon, creating punctures that acquire, apart from the spin $j_{i}$ endowing it with area, new quantum numbers $m_{i}$ (responsible for its intrinsic geometry and such that $\left.-\left|j_{i}\right| \leq m_{i} \leq\left|j_{i}\right|\right)$. The numbers $\left(j_{i}, m_{i}\right)$ can be thought of as the analogues of the total angular momentum and projection along an axis, respectively. These horizon degrees of freedom fluctuate independently of the bulk degrees of freedom and give raise then to the entropy of the horizon. There is also an important issue regarding this formalism. LQG possesses a one parameter family of inequivalent representations of the classical theory labeled by a real number $\gamma$, the so called Barbero-Immirzi (BI) parameter that is absent classically. The strategy is to chose the value of $\gamma$ in such a way that, for large black holes, the entropy corresponds to $(1 / 4$ of $)$ the area in Planck units [5]. 
There is, however, an obvious inconsistency between loop quantum gravity and Bekenstein's considerations: the area spectrum in LQG is not evenly spaced. On the contrary, the LQG area spectrum is given by,

$$
A=\sum_{i} 8 \pi \gamma \ell_{p}^{2} \sqrt{j_{i}\left(j_{i}+1\right)},
$$

where $j_{i}$ are semi-integers and the sum is taken over all the punctures $i$ at the horizon. The spectrum (3) is not only not equidistant, but it can be expected that the eigenvalues accumulate for values of $A$ large in Planck units, given they do for the general area spectrum [6].

The inconsistency between loop quantum gravity and Bekenstein's heuristic arguments seemed to become less relevant when Dreyer noted [7] that LQG might also be consistent with the constraints imposed by asymptotically damped quasinormal modes, as Hod had previously conjectured [8] within Bekenstein's formalism. The idea is that the asymptotic frequency of these classical modes would correspond to the energy of horizon quanta through the standard relation $E=\hbar \omega$. This requirement would then imply that, in the Bekenstein approach, black holes have an equidistant spectrum given by

$$
A=4 \ell_{p}^{2} \ln (3) n,
$$

whereas, in the LQG approach, a minimum area gap, associated to the quantum transition, would be given by $a_{0}=4 \ell_{p}^{2} \ln (3)$. [This requirement implies a particular choice of the Barbero-Immirzi parameter $\gamma$ involving $\ln (3)$.] Even when not fully consistent (area spectra continues to be different), the appearance of a $\ln (3)$ factor seemed to be more than just a coincidence. This initial expectation was however diminished when it was shown that the entropy calculation in LQG gave a different proportionality factor between entropy and area that called for a different value of the Immirzi parameter that was no longer compatible with Hod's considerations [9] (see also [10]).

The purpose of this Letter is to show that there is indeed a deep relation between entropy within the LQG formalism and Bekenstein's heuristic picture (supplemented by Hod's conjectures), even when the relation is much more subtle than it was originally conceived. To be precise, we shall show that a detailed analysis of the number of states compatible with the macroscopic conditions imposed on small, Planck size black holes within the LQG approach yields, when appropriately interpreted, a functional form of the entropy as function of horizon area that realizes in a precise manner Bekenstein's picture. The coincidence turns out to be not only qualitative, but it also incorporates two numbers that are important for both formalisms, namely, $\ln (3)$ and the value $\gamma_{0}$ of the Immirzi parameter (that recovers the Bekenstein-Hawking relation $S=A / 4$ for large black holes).
We have computed the number of states compatible with a horizon of area $A_{0}$ using the formalism developed in [5], that specifies which states have to be counted. We performed the counting using a simple algorithm described in detail in [11]. In the entropy computation within the microcanonical ensemble, one resorts to the usual prescription of counting states whose area eigenvalues $A=\langle\hat{A}\rangle$ lie in an interval $\left[A_{0}-\delta A, A_{0}+\delta A\right]$, and where a total projection constraint $\sum_{i} m_{i}=0$ is imposed such that the horizon geometry is the quantum version of an isolated horizon [5]. The parameter $\delta A$ that fixes the interval is normally assumed to be of the order of Planck area. In [11] it was shown that the entropy, as function of area $A$ has some oscillatory behavior, whose amplitude depends on $\delta A$ but with a constant periodicity that is independent of $\delta A$. Here we have taken further the analysis of [11] in order to unravel the structure of these oscillations. As a first step we have taken a rather small interval $\widetilde{\delta A}=0.005$ (in Planck units) with a point separation of 0.01 , in order to isolate the "spectrum" of the quantum black hole. Note that with this choice, one is covering the full set of values of area, without the intervals overlapping, and what one is doing is to separate the total number of black hole states in different ranges of area, as is done when drawing a histogram. The resulting number is not then employed to determine the entropy (for which a much longer $\delta A$ is employed). The results are plotted in Figs. 1 and 2. The oscillatory behavior found in the entropy [11] as well as the patterns shown in these figures have a period of $\Delta A_{0}=$ $2.41 \ell_{p}^{2}$, approximately.

The next step was to compute the entropy by counting the number of states within a given interval of area, with the choice that the size of the interval coincides with the periodicity of the oscillations, namely, $2 \delta A=\Delta A_{0}$. The

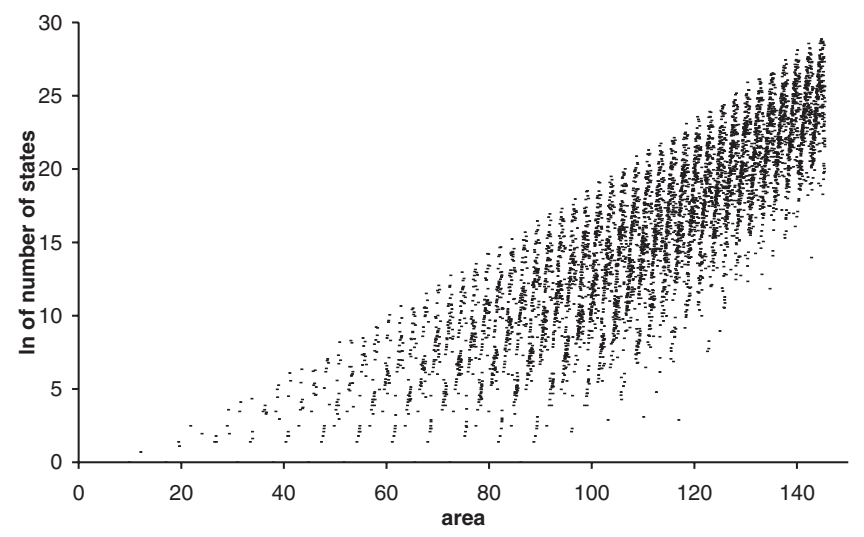

FIG. 1. The (ln of the) number of states as a function of area is shown. The Barbero-Immirzi parameter is taken as $\gamma=0.274$ from $[10,11]$. The interval $\left[A_{0}-\widetilde{\delta A}, A_{0}+\widetilde{\delta A}\right]$ is taken to be rather small $\left(\widetilde{\delta A}=0.005 \ell_{p}^{2}\right)$ so that one is effectively counting the number of states as function of area. The area is shown in Planck units. Note that this does not represent the entropy, given that the interval is very small in Planck units. 


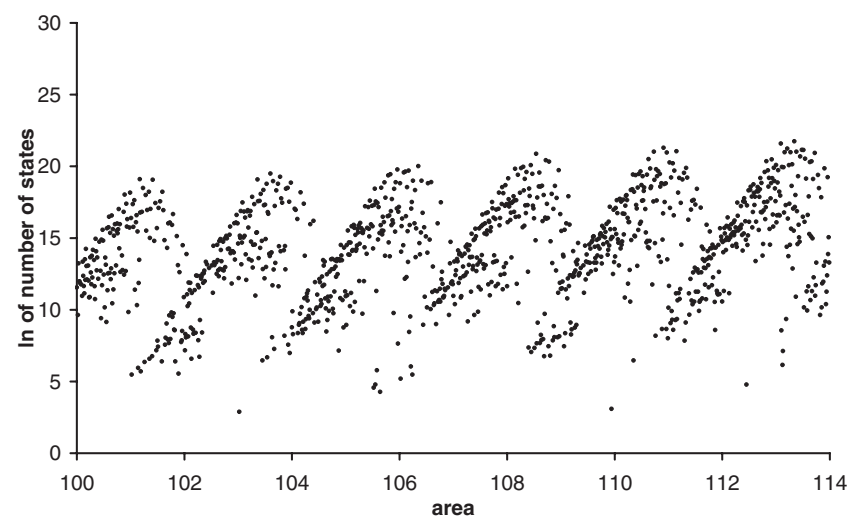

FIG. 2. The same as Fig. 1 but more detail is shown.

resulting entropy is plotted in Figs. 3 and 4, where more details can be appreciated.

Let us now discuss the results. From Figs. 1 and 2 it is clear that the spectrum of the quantum black hole has some new and nontrivial features. Specially noteworthy is the periodic structure that arises when looking at this rather small scale (recall that each Planck area is covered by 100 intervals and thus correspond to 100 points in the graph). The appearance of these "mountain like" structures, that are also periodic with the same period as the oscillations could not have been inferred from the oscillations in the entropy function. Thus, the periodicity of the entropy-area relation has to be associated with these new structures in the spectrum and not with other features such as the change in the number of punctures, a simple transition involving creation/annihilation of edges puncturing the horizon, or any other "naive" explanation of that sort. It is certainly intriguing that this new length scale appears, that as we would like to emphasize, is not related to any other scale previously found in LQG.

Motivated by these considerations, it was natural to explore the entropy counting with an area interval $\delta A$ given by this new scale $2 \delta A=\Delta A_{0}$. The results shown in Figs. 3 and 4 are quite unexpected. The oscillations that are found for all other values of $\delta A$ disappear and instead, one is left with a "ladder" in the entropy vs area graph.

The first conclusion from this graph, is that if one interprets the (ln of the) number of states as physical entropy then there are regions where the area changes but the entropy remains constant. Any quantum transition between those states would then correspond, in a precise sense, to an adiabatic process. Furthermore, we see that entropy (and not area) has effectively only a discrete number of possible values it can take. This is precisely the conclusion that one can draw from Bekenstein's argument, namely, that entropy should be equidistant for large black holes. Even when it can not be fully appreciated from the figures, what we observe is that the ladder is not completely regular for small black holes; the height of the ladder seems to increase, as the black holes grows,

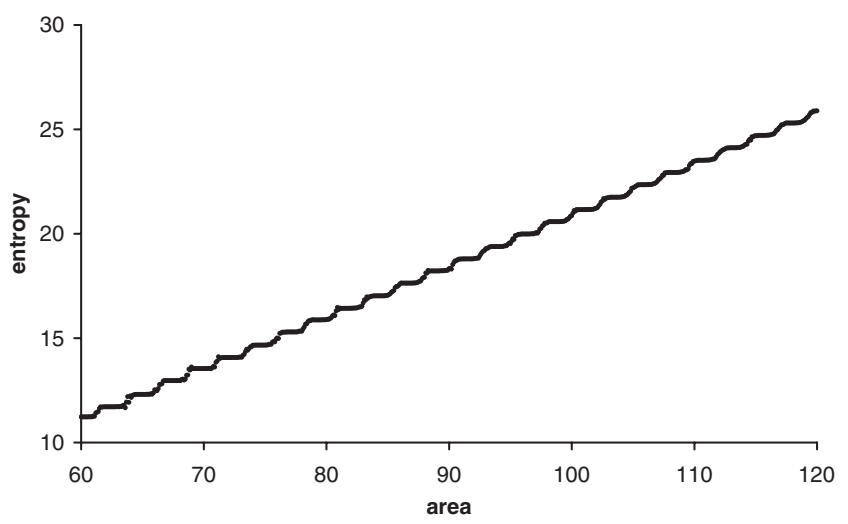

FIG. 3. The entropy as a function of area is shown, where the projection constraint has been imposed, the Barbero-Immirzi parameter is taken as $\gamma=0.274$ and $\delta A$ is taken to coincide with $1 / 2$ of the period of the oscillations in the number of states $\Delta A_{0}$.

approaching a constant value for larger black holes. Thus, what we see is an emergent picture for small black holes within LQG that is consistent with Bekenstein's model. Furthermore, the manner in which the discrete equidistant values emerge is much more subtle than just assuming an equidistant area spectrum. From our perspective, it is a rather nontrivial result that loop quantum gravity does accommodate Bekenstein's picture for quantum black holes in such a subtle way. This is the main result of this Letter.

In order to study the dependence of the period of both area and entropy on the value of the Barbero-Immirzi parameter $\gamma$, we performed a series of runs of the code with different values of the parameter $\gamma$. For the area, we found that the period is indeed linearly dependent with $\gamma$ as has the following (conjectured) dependence:

$$
\Delta A \approx 8 \gamma l_{p}^{2} \ln (3) \text {. }
$$

The plot in Figs. 3 and 4 were drawn for the value $\gamma_{0} \approx$ $0.274 \ldots$ of the parameter that reproduces the BekensteinHawking relation $S=A / 4$ in the large area limit (see

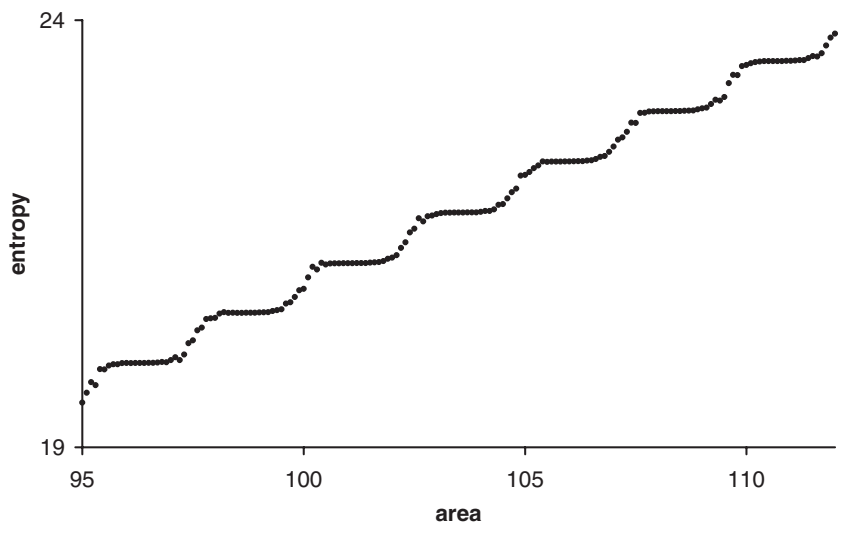

FIG. 4. Same as Fig. 3 but more detail is shown. 
$[10,11]$ for details). The fact that the periodicity in area depends on the value of the Barbero-Immirzi parameter is not surprising since the operator and therefore its eigenvalues depend on it.

For the entropy, we have made the same estimations and the result is somewhat intriguing: the asymptotic size of the steps found in the entropy do not seem to depend on the value of the Barbero-Immirzi parameter $\gamma$. That is, if the conjectured numerical value of the area scale (4) is true, then what we find is a universal value in which the entropy is quantized, namely,

$$
\Delta S \approx 2 \gamma_{0} \ln (3) .
$$

It is certainly remarkable that, as black holes become large, entropy seems to be quantized in integer units of a quantity that contains both "key" numbers: for the heuristic Bekenstein model, $\ln (3)$, and for loop quantum gravity, the value $\gamma_{0}$ of the BI parameter. The precise form of the entropy spectrum is slightly different from Eq. (2) [where $\Delta S=\ln (3)]$, but one should also be aware that the relation (2) was arrived at by means of plausibility arguments rather than a hard core derivation. The conjectured entropy quantization condition derived from (5) is the second result of this Letter.

The main features we have found here about the quantum horizon system, namely, the existence of a pattern in the black hole spectrum with a periodicity that permeates to the entropy-area relation, and the appearance of a new scale associated with this period, could in principle be "generic." That is, one might imagine that these features are common to many quantum systems with a finite number of degrees of freedom. In order to rule out this possibility we have repeated the analysis for a quantum horizon in which the area spectrum is equidistant and given by $A^{\prime}=8 \pi \gamma \ell_{p}^{2} \sum_{i} j_{i}$ (an operator that has been suggested within LQG as well). This would also correspond to the case (modulo a constant) of $N$ decoupled harmonic oscillators in the micro-canonical ensemble. Perhaps not unexpectedly, we have seen that the black hole spectrum is in this case equidistant with an area separation of $\Delta A^{\prime}=$ $8 \pi \gamma \ell_{p}^{2}$, which corresponds to the increase in area when one adds a couple of punctures (the projection constraint $\sum_{i} m_{i}=0$ prevents one from having an odd number of punctures that have the minimum allowed spin, namely, $1 / 2$ ). There is no nontrivial periodic patterns in the spec- trum and the entropy has discrete jumps that are directly associated to the fact that the area spectrum is equidistant.

Another possibility is that this behavior is a consequence of the particular counting procedure used, and that a different one [9] might not have the same properties. We have performed the counting using that procedure and have found the results to be robust: the entropy has discrete jumps and the relations (4) and (5) continue to be valid. Details will be published elsewhere.

We conclude then that the nontriviality of the loop quantum gravity area spectrum (3) is what brings the new and unexpected features to the entropy vs area relation that we have reported in this Letter, and is therefore responsible for black hole entropy quantization. Needless to say, these results can only be a hint of a deeper structure involving gravity, thermodynamics, and the quantum that remains to be unraveled.

We thank A. Ashtekar, J. Lewandowski, and J. NavarroSalas for discussions. This work was in part supported by CONACyT No. U47857-F, No. ESP2005-07714-C03-01, and No. FIS2005-02761 (MEC) Grants, by NSF No. PHY04-56913, the Eberly Research Funds of Penn State, and by the AMC-FUMEC exchange program. J. D. thanks MEC for support through the FPU (University Personnel Training) program.

*Electronic address: corichi@matmor.unam.mx

[1] J. D. Bekenstein, Phys. Rev. D 7, 2333 (1973); S. W. Hawking, Commun. Math. Phys. 43, 199 (1975).

[2] D. Christodoulou, Phys. Rev. Lett. 25, 1596 (1970).

[3] J. D. Bekenstein, gr-qc/9808028.

[4] J. D. Bekenstein and V. F. Mukhanov, Phys. Lett. B 360, 7 (1995).

[5] A. Ashtekar, J. C. Baez, A. Corichi, and K. Krasnov, Phys. Rev. Lett. 80, 904 (1998); A. Ashtekar, J. C. Baez, and K. Krasnov, Adv. Theor. Math. Phys. 4, 1 (2000).

[6] A. Ashtekar and J. Lewandowski, Classical Quantum Gravity 14, A55 (1997).

[7] O. Dreyer, Phys. Rev. Lett. 90, 081301 (2003).

[8] S. Hod, Phys. Rev. Lett. 81, 4293 (1998).

[9] M. Domagala and J. Lewandowski, Classical Quantum Gravity 21, 5233 (2004); K. A. Meissner, Classical Quantum Gravity 21, 5245 (2004).

[10] A. Ghosh and P. Mitra, Phys. Lett. B 616, 114 (2005).

[11] A. Corichi, J. Díaz, and E. Fernández-Borja, Classical Quantum Gravity 24, 243 (2007). 